\title{
IS RISK TO MOTOR NEURON DISEASE INFLUENCED BY THE SEASON OF BIRTH?
}

\author{
Kiyotaro KonDo ${ }^{1, *}$ and Keiko FuJIKl ${ }^{2}$ \\ ${ }^{1}$ Department of Public Health, Hokkaido University, School of Medicine, \\ Kita-ku, Sapporo 010, Japan \\ 2Department of Ophthalmology, Juntendo Medical College, \\ Bunkyo-ku, Tokyo 113, Japan
}

\begin{abstract}
Summary Months of the birth in 310 cases with motor neuron disease were compared with 244 of their spouses who were used as the controls. Cases were born more often in the spring to summer months and less in the winter months. Birth factors should be carefully evaluated in this disease.
\end{abstract}

Key Words motor neuron disease, seasonal effect, month of birth

\section{INTRODUCTION}

Since neurons are fixed postmitotic cells produced in the early life and age as the host individual ages, it is possible that ante- or perinatal factors predispose the neurons to the process which ultimately manifest as motor neuron disease (MND) after a few decades. In an attempt to have evidences for this possibility, we analyzed vital data of the patients to evaluate effects of the parental age and birth order with essentially negative results (Kondo and Fujiki, 1984). In this report, the same data were further analyzed for possible effect of the season of birth in the pathogenesis of MND.

\section{MATERIALS AND METHODS}

Months of the birth in 310 cases of MND and 244 of their spouses were identified by their Koseki, a legal family register. Methods of collecting cases and the Koseki were described elsewhere (Kondo and Fujiki, 1984). Spouses were considered unbiased with respect to the month of birth and were used as the controls.

The 3-month moving means $(\mathrm{M})$ of the observed monthly number of the cases were calculated. The expected numbers of the cases (E) for each month of birth

Received May 22, 1989; revised version received June 13, 1989; Accepted June 14, 1989.

* To whom correspondence should be addressed. 
were calculated by distributing the observed total, 310 cases, based on the observed moving means for the respective months in the spouses. $M / E$ is 1 if the month of birth has no effects on MND. Sample size did not allow a splitting of the data by sex. Chi-squares between the observed and the expected moving means in each month were totaled.

Kimura and Miura (1983) analyzed 364 cases with amyotrophic lateral sclerosis (ALS) from the Tokyo area which did not overlap with the present cases. Their data were rearranged so that they are comparable with ours.

\section{RESULTS}

In Table 1, total of the chi-squares was 4.8 , being short of $\chi^{2}{ }_{11}(0.05)=19.68$, indicating no significant seasonal effect. The Kolmogorov-Smirnov value was not significant $(p>0.05)$. M/E's tended to be elevated than 1 in the summer months while they were low in the winter months, however. In Table 2, more cases were born from April through July, but less from September to February than predicted, but the trend was insignificant.

Both results were somewhat different, although the peak months of $\mathrm{M} / \mathrm{E}$ 's remained in the spring to summer month.

\section{DISCUSSION}

Neurons are formed early in life and subject to influences of various factors throughout the lifetime.

True cause of MND is still obscure. ALS represents about $80 \%$ of the cases with MND. Fragmentary evidences suggested that MND is influenced by environmental factors including sociocultural factors (Kondo, 1988), but an important problem remains practically untouched mainly because of technical difficulties whether ante- or perinatal factors, through some influences on developing motor neurons, predispose to MND which clinically manifests in later decades of life. Koseki allowed us to analyze this aspect at least from biometrical points of view. In diseases influenced by congenital or perinatal factors, some variables about the birth change according to the pathomechanism. Elevated maternal age in Down's syndrome is an example which is conditioned by the fact that chromosome nondisjunction increases as the ova age.

Our previous work showed no effect of parental age, birth order, sib size as well as birth interval (Kondo and Fujiki, 1984). In the present analyses, we analyzed the effect of month of birth in the same cases, as well as in another set of data collected by other authors. Two groups of cases showed modest excess of cases who were born in the spring to the summer months, but the trends were insignificant. Whether some season-dependent factors in early life influence subsequent MND awaits further confirmation. Since the season is reverted, it is useful to repeat a similar analysis in the Southern hemisphere to evaluate the present hypothesis. 

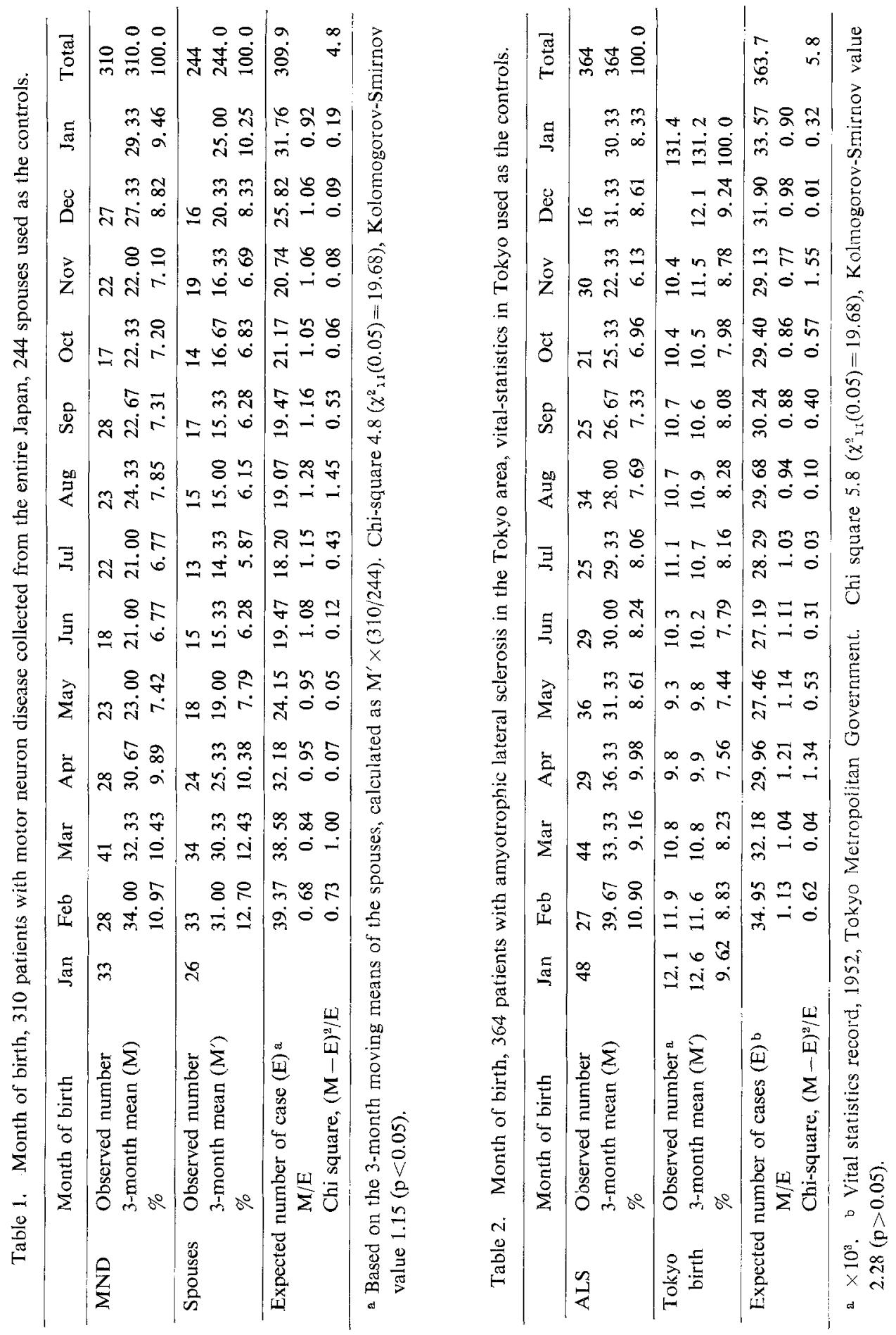
Supported by a grant for the Neurodegenerative Disease, the Japan Ministry of Health and Welfare.

\section{REFERENCES}

Kimura, T. and Miura, T. 1983. Multiple sclerosis, amyotrophic lateral sclerosis and birth season. Med. Biol. Jpn. 107: 77-80.

Kondo, K. 1988. Epidemiology of motor neuron disease in Japan: declining trends of the mortality rate. In Amyotrophic Lateral Sclerosis, Tsubaki, T. and Yase, Y., eds, Elsevier, Amsterdam, pp. 11-16.

Kondo, K. and Tsubaki, T. 1977. Changing mortality patterns of motor neuron disease in Japan. J. Neurol. Sci. 32: 411-424.

Kondo, K. and Fujiki, K. (1984). Effects of parental age and birth order in motor neuron disease. Jpn. J. Human Genet. $29:$ 45-50. 